\title{
Large-Scale 3D Modeling of Projectile Impact Damage in Brittle Plates
}

\author{
A. Seagraves ${ }^{a}$, R. Radovitzky ${ }^{b}$ \\ ${ }^{a}$ Department of Mechanical Engineering \\ Institute for Soldier Nanotechnologies \\ Massachusetts Institute of Technology \\ Cambridge, MA 02139 \\ ${ }^{b}$ Department of Aeronautics and Astronautics \\ Institute for Soldier Nanotechnologies \\ Massachusetts Institute of Technology \\ Cambridge, MA 02139
}

\begin{abstract}
The damage and failure of brittle plates subjected to projectile impact is investigated through large-scale three-dimensional simulation using the DG/CZM approach introduced in [1]. First, we simulate edge-on impact experiments on $\mathrm{Al}_{2} \mathrm{O}_{3}$ tiles (Strassburger and Senf, ARL Tech Report). Qualitative and quantitative validation of the simulation results is pursued by direct comparison of simulations with experiments at different loading rates and good agreement is obtained. In the second example considered, we investigate the fracture patterns in normal impact of spheres on thin, unconfined ceramic plates over a wide range of loading rates. For both the edge-on and normal impact configurations, the full field description provided by the simulations is used to interpret the mechanisms underlying the crack propagation patterns and their strong dependence on loading rate.
\end{abstract}

Keywords: 


\section{Introduction}

The damage and failure of brittle materials subjected to impact loads is characterized by the development of intricate patterns of three-dimensional cracks. In localized impact loading, cracks are driven by complex dynamic stress fields which arise as a result of stress wave propagation. These dynamic stresses can be highly complex and heterogeneous due to the interaction of stress waves with free boundaries, material interfaces, and newly formed crack surfaces $[2,3,4,1]$. Improving the fundamental understanding of how dynamic stress fields drive three-dimensional brittle damage patterns is critical in many applications including armor materials [5, 6], orbital debris mitigation [7] and hydraulic fracturing [8].

In order to gain insight into this problem, there has been a strong effort over the last fourty years to study impact damage through plate impact experiments where the cracking patterns were analyzed in recovered specimens $[2,3,9,10,11,12,13,14,15,16,17,18,19,20]$. A key finding of these studies is that although highly complex, the cracking patterns resulting from impact loads can sometimes be decomposed into a small set of characteristic fracture modes whose propagation paths follow simple trajectories. For example, some of the characteristic cracking patterns have a rotational symmetry about the impact axis. The most common examples of axisymmetric cracking patterns are "conical" and "ring" cracks.

Conical cracks nucleate near the tile surface directly adjacent to the contact zone and propagate into the volume along conoidal fronts which are characterized by a cone angle (see Figure 1). Another axisymmetric cracking mode that is observed frequently in impact experiments are "ring" cracks, 
which are circular cracking patterns that nucleate some distance outside of the contact zone on the impacted tile surface (see Figure 2). Ring cracks are characterized by their average radius which is measured from the central impact point. Both conical and ring cracks are ubiquitous features of brittle impact damage and have been observed in impact experiments on a variety of different brittle materials including glasses $[9,12]$, nominally brittle polymers [18], and ceramics $[2,3,10,11,13,14,15,16,17,19,21,20]$.

Axisymmetric crack fronts occur in brittle materials impacted by bodies of revolution because the dynamic stress fields that drive these cracking patterns have a corresponding rotational symmetry about the impact axis. In addition to providing the driving force for nucleation of axisymmetric crack fronts, the rotationally symmetric stress fields in an impacted plate also drive a symmetry-breaking characteristic cracking mode which is inherently three-dimensional. This failure mode, known as "radial" cracking, is characterized by multiple planar cracks propagating simultaneously in radial directions from the impact region. Similarly to the axisymmetric fracture modes, radial cracks have been observed in impact experiments on a variety of brittle materials $[3,10,12,13,14,15,16,5,18,21]$. Figure 3 shows a typical post-mortem radial cracking pattern on the back face of a damaged $\mathrm{Al}_{2} \mathrm{O}_{3}$ tile after impact by a steel sphere at $586 \mathrm{~m} / \mathrm{s}$ from [22].

The nucleation and propagation of radial cracking patterns is generally attributed to hoop stress fields which arise at some depth directly below the impact point $[3,15,16]$. Correspondingly, radial cracks have been observed to nucleate well below the impacted surface either in the specimen volume [3], or on the opposing back surface of the plate. The plate back surface can 


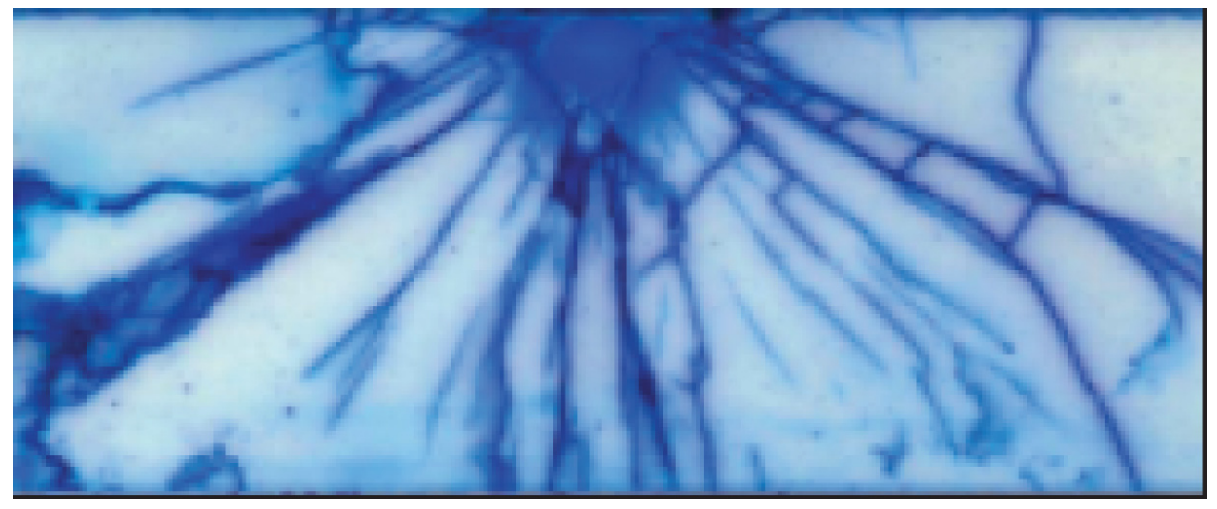

(a) $\mathrm{Al}_{2} \mathrm{O}_{3}$ specimen

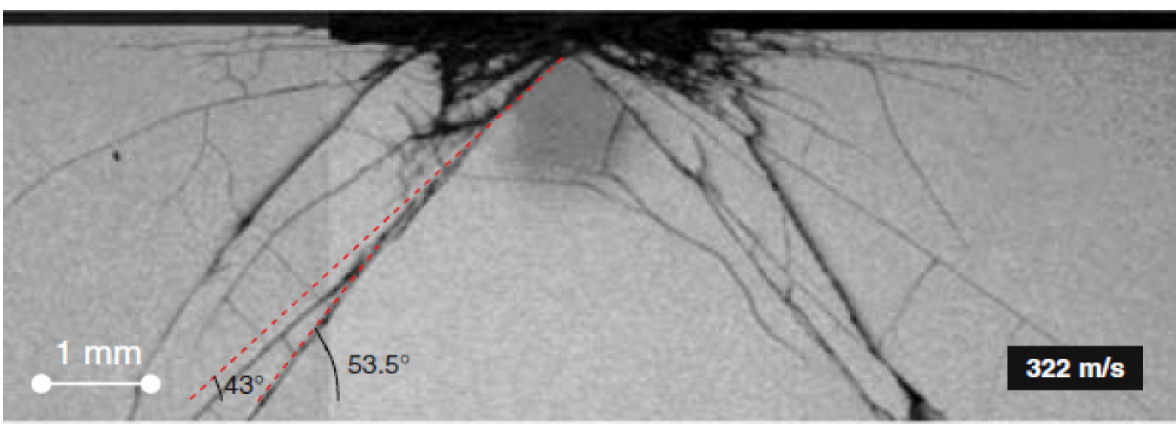

(b) $\mathrm{SiC}$ specimen

Figure 1: Post-mortem cross sectional views of crack patterns in ceramics tiles impacted by spheres. a) $\mathrm{Al}_{2} \mathrm{O}_{3}$ tile, 304 Stainless steel sphere, $586 \mathrm{~m} / \mathrm{s}$ impact velocity [20], and b) $\mathrm{SiC}$ tile, WC sphere, $322 \mathrm{~m} / \mathrm{s}$ impact velocity [19]. 




Figure 2: Ring cracks on the surface of an SiC specimen impacted by a steel sphere at 270 $\mathrm{m} / \mathrm{s}[11]$

be either a free boundary for unsupported plates [12, 14, 18], or a material interface for a plate with a backing support $[13,15,16,5,21]$. Radial cracks greatly complicate the analysis of damage in impact scenarios because of their tendency to nucleate in subsurface regions and because they require a fully three-dimensional analysis for a problem that could otherwise be studied in two dimensional axisymmetric mode.

Rotational symmetry of the damage response can also be violated when stress waves reflect from the lateral boundaries of the specimen. Boundary reflections can cause non-axisymmetric cracking to occur from localized tensile stresses in the reflected waves, or by the interaction of reflected waves with propagating cracks. These symmetry-breaking boundary effects are strongest when the lateral boundaries of the specimen are free surfaces. Stress wave reflections at free lateral boundaries not only promote an inherently threedimensional damage response, but can also cause complete fragmentation of an impacted sample, as observed by Sherman in several experimental studies 


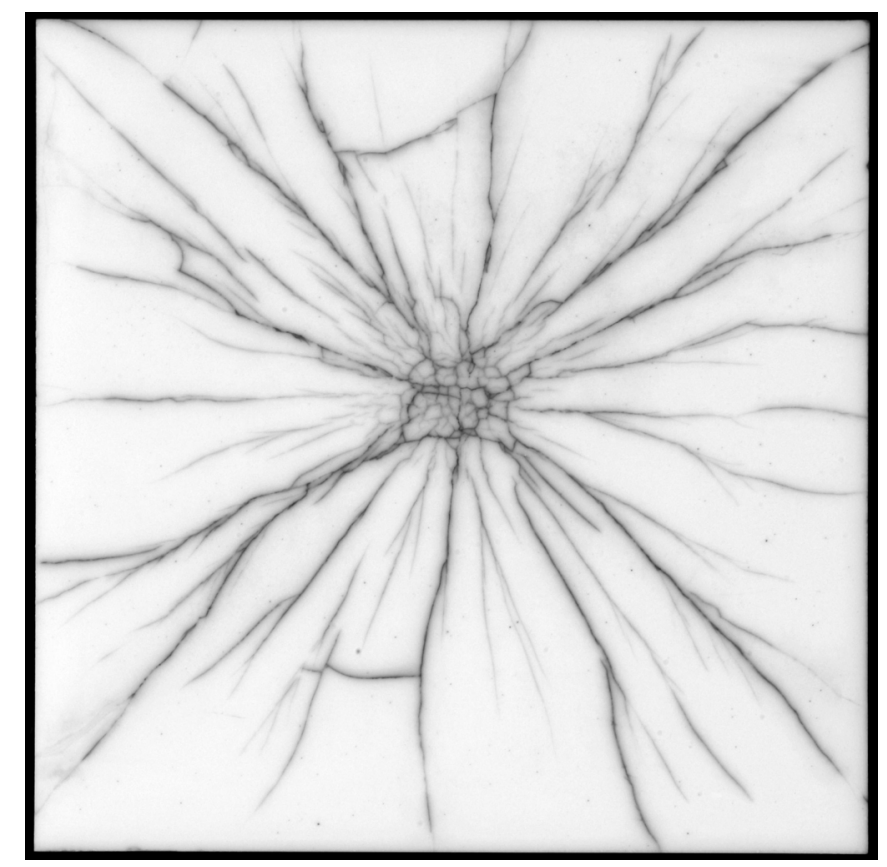

Figure 3: Radial cracking patterns on the back face of an $\mathrm{Al}_{2} \mathrm{O}_{3}$ tile impacted by a steel sphere at $586 \mathrm{~m} / \mathrm{s}$ from $[22]$ 
of high speed projectile impact on $\mathrm{Al}_{2} \mathrm{O}_{3}$ tiles $[13,14,15,16]$.

The large body of experimental evidence collected in plate impact studies shows that the progressive development of damage in an impacted plate will generally entail the nucleation and propagation of some combination of axisymmetric and non-axisymmetric cracking modes. The type of failure modes that are activated, their sequence of activation and subsequent interaction, and the final overall fracture pattern depends strongly on target and projectile material properties, target geometry and boundary constraints, and projectile speed $[2,3,10,12,13,15,16,17]$. This parametric dependence has been studied experimentally to some extent. For example, Evans and Wilshaw [3] conducted impact experiments of WC spheres on semi-infinite ZnS plates at different impact velocities. In their tests, the authors observed the trace of radial cracks on the impacted surface along with fragmentation and material removal from the plate surface. Their rather intuitive results indicated that both the average final radial crack lengths and the extent of material removal from the impact surface increased with impact velocity.

Similar results were reported by Normandia [17] in experiments of WC sphere impact on thin SiC-N plates encased in a steel confinement chamber. For impact velocities up to $393 \mathrm{~m} \cdot \mathrm{s}^{-1}$, only radial cracks were nucleated. Above this threshold velocity, a fragmented crater developed under the penetrator accompanied by radial cracks, ring cracks and material removal from a shallow fragmented circumferential zone at the impacted surface. Normandia measured the radial extent of the fragmented region on the top surface and found that it increased linearly with impact velocity. Ball and McKenzie [12] investigated the effects of impact velocity and specimen thickness on 
the fracture patterns in glass plates impacted by steel spheres. The authors observed radial, ring, and conical cracks and crushing of material under the penetrator and found that the particular combination of fracture modes for a given test depended sensitively on both the specimen thickness and the impact velocity.

In a series of papers $[13,14,15,16]$, Sherman and co-workers conducted a comprehensive parametric study of high velocity impact on $\mathrm{Al}_{2} \mathrm{O}_{3}$ tiles. An important finding from these tests is that the predicted fracture response depends strongly on the tile thickness and on the confinement conditions at the lateral boundaries of the specimen. In $[15,16]$, Sherman used steel and aluminum supporting plates which showed traces of plastic deformation in coincidence with the location of radial cracks. This provided for the first time, a quantitative correlation between test parameters and the number of radial cracks and showed that the number of cracks depends strongly on the tile thickness. Sherman also studied the effects of lateral confinement in [14, $15,16]$ and found that tiles with free lateral boundaries sustained a greater amount of damage than confined tiles under equivalent impact conditions. Indeed, while many of the confined tiles were recovered intact, the tiles with free lateral boundaries were completely fragmented in all cases. Sherman attributed the severity of damage in unconfined tiles to the activation of additional failure modes from stress waves reflected at the lateral specimen boundaries.

While these experimental studies have provided some key insights into the complex dependence of cracking patterns on the problem parameters in projectile impact scenarios, some fundamental questions still remain unan- 
swered. For example, what aspects of the stress field control the activation and evolution of different fracture modes and how are the stresses driving damage affected by changes in problem parameters such as the impact speed and the tile thickness as well as by the propagating cracks? Also, what is the influence of non-axisymmetric boundary effects as a function of problem parameters and under what conditions are boundary effects important?

In this paper, we attempt to address these questions through large-scale three-dimensional simulation of dynamic fracture in brittle plates subjected to high velocity impact. We employ the new computational approach introduced in [1] which is based on combining discontinuous Galerkin methods with cohesive zone models of fracture (called DG/CZM). The goal of this study is to examine how changes in problem parameters affect the evolution of the driving stresses for damage formation and correspondingly, the activation, evolution, and interaction of different failure modes. Particular emphasis is placed on studying the effects of parameter variations on nonaxisymmetric aspects of the tile response, such as radial cracks and damage caused by stress waves reflected from the specimen boundaries. This is in contrast to most of the previous computational work in modeling projectile impact damage which has mainly focused on studying damage development in two-dimensional axisymmetric mode $[23,24,25,26,4,27,28,20]$. In [1], we demonstrated that the DG/CZM method is able to represent conical and radial cracking patterns. In this paper we emphasize the comparison of simulations to experiments and in interpreting the simulations to infer the mechanisms of fracture.

The outline of this paper is as follows. In section 2, we briefly summarize 
the DG/CZM numerical framework. In section 3.1 we apply the method to simulate edge-on impact experiments from [29] and we make detailed comparisons between simulations and experimental results. Then in section 3.2 we simulate the normal impact of rigid spheres on thin, unconfined ceramic tiles. For both the edge-on and normal impact configurations, we investigate the dependence of the predicted fracture patterns on impact velocity through parametric simulation studies. Through detailed visualization and analysis of the stress field evolution we attempt to identify basic mechanisms underlying impact velocity dependence of the cracking patterns.

\section{DG/CZM Computational Framework}

\subsection{Finite Element Framework}

The basic idea underlying the DG/CZM finite element framework developed in [1] is that discontinuous displacement jumps are permitted at all element boundaries from the beginning of the calculation. In the uncracked continuum, $C^{0}$ continuity at the inter-element boundaries is enforced weakly, leading to an elementwise-continuous polynomial approximation $\boldsymbol{\varphi}_{h}$

of the deformation over the discretization $B_{0 h}=\bigcup_{e=1}^{E} \bar{\Omega}_{0}^{e}$ of a body $B_{0}$. The assumption of a discontinuous displacement field then gives rise to additional inter-element boundary integrals in the weak statement of the problem which guarantee consistency and stability of the finite element solution [30, 1]. These inter-element integrals are computed through the use of interface elements in a manner similar to extrinsic cohesive element methods based on the continuous Galerkin framework $[4,31]$. At each time step in the calculation, a fracture criterion is checked at each interface element quadrature point. 
When fracture is detected, the DG inter-element or flux terms are replaced with the virtual work of the cohesive tractions, effectively creating new crack surface. Specifically, the weak formulation of the DG/CZM problem can be stated as:

$$
\begin{aligned}
& \int_{B_{0 h}}\left(\rho_{0} \ddot{\boldsymbol{\varphi}}_{h} \cdot \delta \boldsymbol{\varphi}_{h}+\mathbf{P}_{h}: \nabla_{0} \delta \boldsymbol{\varphi}_{h}\right) d V+ \\
& \underbrace{\int_{\partial_{I} B_{0 h}}(1-\alpha) \llbracket \delta \boldsymbol{\varphi}_{h} \rrbracket \cdot\left\langle\mathbf{P}_{h}\right\rangle \cdot \mathbf{N}^{-} d S}_{\text {DG consistency term }}+ \\
& \underbrace{\int_{\partial_{I} B_{0 h}}(1-\alpha) \llbracket \delta \boldsymbol{\varphi}_{h} \rrbracket \otimes \mathbf{N}^{-}:\left\langle\frac{\beta_{s}}{h_{s}} \mathbb{C}\right\rangle: \llbracket \boldsymbol{\varphi}_{h} \rrbracket \otimes \mathbf{N}^{-} d S}_{\text {DG stability term }}+ \\
& \underbrace{\int_{\partial_{I} B_{0 h}} \alpha \mathbf{T}\left(\llbracket \boldsymbol{\varphi}_{h} \rrbracket\right) \cdot \llbracket \delta \boldsymbol{\varphi}_{h} \rrbracket d S}_{\text {Cohesive law term }} \\
& =\int_{B_{0 h}} \rho_{0} \mathbf{B} \cdot \delta \boldsymbol{\varphi}_{h} d V+\int_{\partial_{N} B_{0 h}} \overline{\mathbf{T}} \cdot \delta \boldsymbol{\varphi}_{h} d S
\end{aligned}
$$

The first integral on the left hand side along with the two integrals on the right hand side of Equation (1) constitute the classical continuous Galerkin (CG) formulation where $\rho_{0}$ is the mass density per unit reference volume, $\mathbf{B}$ is the body force per unit mass and $\overline{\mathbf{T}}$ are external surface tractions applied on the Neumann boundary, $\partial_{N} B_{0} . \mathbf{P}_{h}$ is the first Piola-Kirchhoff stress tensor, and $\delta \varphi_{h}$ is a suitable test function.

The last three integral terms on the left hand side of Equation (1) represent the inter-element contributions arising from the DG and cohesive zone model formulations. The first two inter-element integrals are the DG terms which result from the weak enforcement of inter-element continuity in the pre-fracture regime, defined by $\alpha=0$. These integrals ensure consistency 
and stability of the solution in the presence of discontinuous displacement fields in the uncracked continuum as indicated in Equation (1) [30]. In these expressions, $\llbracket \bullet \rrbracket=\left[\bullet^{+}-\bullet^{-}\right]$and $\langle\bullet\rangle=\frac{1}{2}\left[\bullet^{+}+\bullet^{-}\right]$are the jump and average operators, $\mathbf{N}^{-}$is the outward unit reference normal to the minus side of an interface element (see Figure 4$), \beta_{s}$ is a stability parameter, $h_{s}$ is a characteristic element size, and $\mathbb{C}=\frac{\partial \mathbf{P}}{\partial \mathbf{F}}$ are the Lagrangian tangent moduli. After fracture is detected at an interface element quadrature point, $\alpha$ is set to 1 and the third term, representing the contribution of the cohesive traction separation law $\mathbf{T}\left(\llbracket \boldsymbol{\varphi}_{h} \rrbracket\right)$, becomes operative.

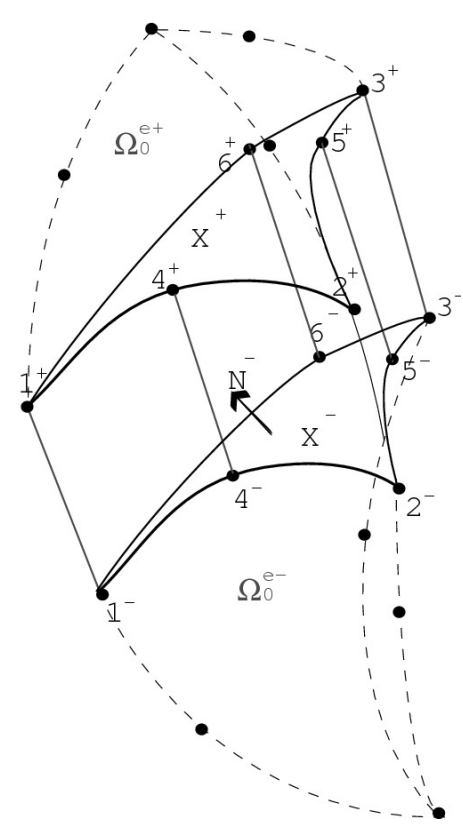

Figure 4: Description of a 12-node interface element introduced between two 10-node quadratic tetrahedra $\Omega_{0}^{e+}$ and $\Omega_{0}^{e-}$.

A key advantage of DG/CZM is that the method resolves the well-known issue of "artificial compliance" in intrinsic cohesive element methods $[32,33]$ 
in which wave characteristics in the uncracked continuum are affected by the presence of inter-element discontinuities in the pre-fracture regime. The ability of the DG/CZM to accurately describe stress wave propagation is critical for modeling damage in impact problems as the propagation of stress waves plays a key role in driving the nucleation, propagation, and arrest of sharp cracks. Another advantage of DG/CZM, is that the parallel implementation in three dimensions is highly scalable [1]. This is in contrast to three-dimensional implementations of the extrinsic cohesive element method, described in $[34,35,36]$, which require on-the-fly modification of the finite element mesh topology, a complex process which has hindered the development of scalable algorithms in three dimensions. This capability provides a unique opportunity to investigate complex dynamic fracture patterns in three dimensions. Extensive details on the finite element implementation of the DG/CZM method and its parallelization and scalability up to thousands of processors can be found in [1].

\subsection{Cohesive Law}

The description of the DG/CZM method is completed by choosing a particular form for the cohesive law. In this work, we adopt the irreversible, linear softening cohesive law of [31]. This particular TSL assumes that the cohesive behavior is isothermal, isotropic, and that the cohesive tractions depend on the local state of deformation at the crack tip only through the displacement jump. A simple formulation for modeling mixed-mode fracture, in accordance with these assumptions, is obtained by assuming that the cohesive free energy density depends on the surface opening vector $\boldsymbol{\Delta}$ only 
through an effective separation $\delta$ defined by

$$
\delta=\sqrt{\gamma^{2} \Delta_{m}^{2}+\Delta_{n}^{2}}
$$

In this expression, $\Delta_{n}$ is the positive normal separation along the unit local normal in the deformed configuration $\mathbf{n}, \Delta_{m}$ is the tangential separation along the unit local tangent in the deformed configuration $\mathbf{m}$, and $\gamma$ is a parameter which assigns different weights to normal and tangential separations.

Under this set of assumptions, one can define an effective cohesive traction $T$ per unit undeformed area which is given by

$$
T=T(\delta, \mathbf{q})
$$

where $\mathbf{q}$ is a suitable set of internal variables describing the irreversible processes involved in decohesion. This law becomes operative at a quadrature point of an interface element when the fracture criterion

$$
\sqrt{\left(\boldsymbol{\sigma}_{h}:[\mathbf{n} \otimes \mathbf{n}]\right)^{2}+\gamma^{-2}\left(\boldsymbol{\sigma}_{h}:[\mathbf{n} \otimes \mathbf{m}]\right)^{2}} \geq \sigma_{c}
$$

is satisfied. In this expression, $\boldsymbol{\sigma}_{h}$ is the Cauchy stress, and $\sigma_{c}$ is the critical effective cohesive strength. Once the effective traction is determined, the components of the traction vector follow from

$$
\mathbf{T}=\frac{T}{\delta}\left(\gamma^{2} \Delta_{m} \mathbf{m}+\Delta_{n} \mathbf{n}\right)
$$

In the specific case of the linear irreversible softening law, the functional form of the effective cohesive traction for crack opening is given by

$$
T\left(\delta, \delta_{\max }\right)=\sigma_{c}\left(1-\frac{\delta}{\delta_{c}}\right) \frac{d s}{d S} \quad \text { for } \dot{\delta} \geq 0, \delta=\delta_{\max }
$$


where $\frac{d s}{d S}$ is the surface change between the deformed and reference configuration. Complete decohesion $(T \equiv 0)$ occurs when the maximum effective opening displacement $\delta_{\max }$ exceeds a critical value $\delta_{c}$. $\delta_{\max }$ constitutes the internal variable describing irreversibility. For crack unloading, characterized by $\dot{\delta}<0$ or $\delta<\delta_{\max }$, the functional form of the linear irreversible softening law is assumed to follow a straight path to and from the origin yielding

$$
T\left(\delta, \delta_{\max }\right)=\frac{T_{\max }}{\delta_{\max }} \delta \quad \text { for } \dot{\delta}<0, \text { or } \delta<\delta_{\max }
$$

where $T_{\max }$ is the value of the effective traction at $\delta=\delta_{\max }$. The work of separation (denoted by $\phi_{\mathrm{s}}$ ) for the linear softening law is simply

$$
\phi_{\mathrm{s}}=\frac{1}{2} \sigma_{c} \delta_{c} \frac{d s}{d S}=G_{c},
$$

where $G_{c}$ is the fracture energy, which can be obtained from experiments.

For crack closure, i.e. $\Delta_{n}=0$ and $\dot{\Delta}_{n}<0$, we assume that the normal contact stresses follow the continuum prescription, and therefore fall back to the DG form of the interface terms. This guarantees that compressive stress wave components can propagate across the closed crack surface as in the uncracked body. The tangential response is still governed by the TSL. Improvements on this model would incorporate a frictional component in the tangential response, but this has been neglected in the present work for simplicity and due to a lack of experimental data supporting this need.

\section{Simulations of Brittle Plate Impact}

\subsection{Edge-On Impact (EOI) Simulations}

In [29], Strassburger and Senf conducted an extensive experimental study of dynamic fracture in thin glass and ceramic plates subjected to edge-on 
impact (EOI) by steel cylinders. In the experiments, high speed video was used to visualize the evolution of cracking patterns on the plate surfaces. The high speed video images revealed that the key failure mechanisms for ceramics are: the propagation of cone cracks from the edges of the contact zone and the development of an expanding damage front composed of many cracks nucleating and propagating along planes directed outwardly in the radial direction. Experiments conducted at different impact velocities showed that the overall dynamic fracture pattern depends strongly on the loading rate. Visualization of the experimental cracking patterns also provided a basis for extracting quantitative information such as the average propagation distance of the radial fracture front over time.

In this section, we present simulations of the edge-on impact experiments from [29] for the case of $\mathrm{Al}_{2} \mathrm{O}_{3}$ tiles subject to a range of different impact velocities. The simulation results are validated by direct comparison with the experiments. The EOI configuration considered in the simulations is depicted in Figure 5. For simplicity, the cylinder is treated as a rigid body in the simulations and contact between the cylinder and the plate is modeled using a simple penalty contact formulation. The finite element mesh, obtained with Gmsh software [37], was optimized to eliminate structure that might bias the predicted crack paths [38]. The mesh containing 266,000 volumetric tetrahedra, or a total of 7.98 million degrees of freedom, is shown in Figure 5. In the simulations, test velocities corresponding to 85 and $150 \mathrm{~m} \cdot \mathrm{s}^{-1}$ are considered. In this relatively low velocity regime, the effects of plasticity can be neglected as the impact velocities are below free surface velocities recorded at the Hugoniot elastic limit in flyer plate experiments on $\mathrm{Al}_{2} \mathrm{O}_{3}$ 


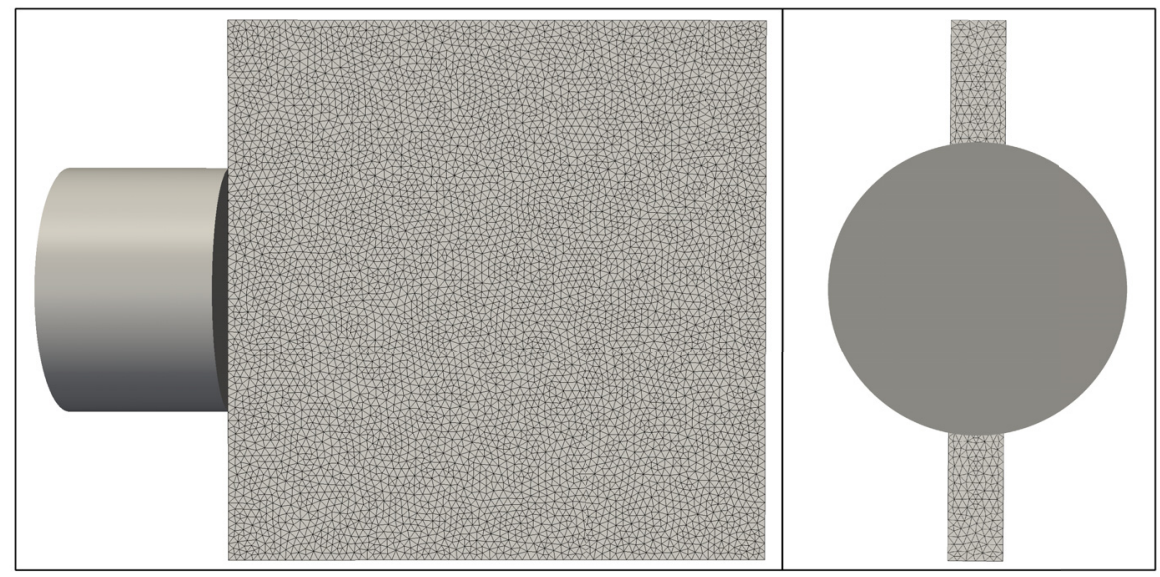

Figure 5: The initial configuration for simulations of the edge-on impact tests of Strassburger and Senf [29]. The figure shows the finite element discretization used in the simulations, consisting of 266,000 volumetric elements.

[39]. As a result, the bulk material is assumed to behave elastically up to the point of fracture. The material properties and problem parameters used in simulations are provided in Table 1.

In Figures 6(a)-6(d), the computed evolution of the crack patterns for $V_{c}=85 \mathrm{~m} \cdot \mathrm{s}^{-1}$ is compared to high speed video images of the experimental observations. In the early stages of the impact event, cone cracks nucleate and propagate into the specimen from the upper and lower edges of the contact zone boundary accompanied by crack nucleation some distance away from the contact zone along the centerline of the impactor. Eventually, cracks nucleate away from the centerline of the impactor which propagate along radial planes, creating a fan-shaped damage front in the specimen. Cracks also form in localized regions on the upper and lower edges of the specimen. The images in Figures 6(a)-6(d) show a good qualitative agreement between 


\begin{tabular}{ll}
\hline Properties & \multicolumn{2}{c}{ Values } \\
\hline \multicolumn{1}{c}{$\mathrm{Al}_{2} \mathrm{O}_{3}$ Plate } \\
\hline Length & $L=10 \mathrm{~cm}$ \\
Thickness & $t=1 \mathrm{~cm}$ \\
Initial density & $\rho_{0}=3690 \mathrm{~kg} \cdot \mathrm{m}^{-3}$ \\
Elastic Modulus & $E=370 \mathrm{GPa}$ \\
Poisson's Ratio & $\nu=0.22$ \\
DG Stability Parameter & $\beta=4$ \\
Cohesive Strength & $\sigma_{c}=400 \mathrm{MPa}$ \\
Fracture Energy & $G_{c}=25 \mathrm{~J} \cdot \mathrm{m}^{-2}$ \\
Tension/Shear Weighting & $\gamma=5$ \\
\hline \multicolumn{2}{c}{ Rigid Cylinder } \\
\hline Radius & $R_{c}=1.5 \mathrm{~cm}$ \\
Mass & $m_{c}=0.126 \mathrm{~kg}$ \\
Velocity & $V_{c}=85,150 \mathrm{~m} \cdot \mathrm{s}^{-1}$ \\
\hline
\end{tabular}

Table 1: Plate and projectile dimensions and material properties used for simulations of the EOI experiments of Strassburger and Senf [29]. 
simulations and experiments for both the spatial and temporal occurrence of crack nucleation and the subsequent crack propagation paths.

Quantitative information describing the crack propagation from simulations for the lower impact velocity was obtained by measuring the furthest extent of the radial damage front along the centerline of the specimen and the length of the upper cone crack over time. The results were compared to the average damage front location and cone crack propagation length reported by Strassburger [29], Figure 7. As the plot shows, the simulated cone crack length and the damage front location are in reasonable quantitative agreement with the experiments. However, the average speeds of propagation for the cone crack and the damage front were 5045 and $6829 \mathrm{~m} \cdot \mathrm{s}^{-1}$, respectively, which are higher than the experimentally reported values of 3860 and 5320 $\mathrm{m} \cdot \mathrm{s}^{-1}$. This can be attributed to uncertainty in material parameters and in the actual locations of the crack tips.

Figures $8(\mathrm{a})-8(\mathrm{e})$ compare the results of simulations and experiments for a cylinder impact at $150 \mathrm{~m} \cdot \mathrm{s}^{-1}$. Similarly to the lower impact velocity case, the simulations capture the propagation of a complex front of cracks traveling in radial directions away from the impact point. However, while the fracture front at $85 \mathrm{~m} \cdot \mathrm{s}^{-1}$ features the propagation of just a few dominant cracks, at $150 \mathrm{~m} \cdot \mathrm{s}^{-1}$ many more cracks are nucleated. Notably, a large number of short, closely-spaced cracks nucleate in between longer, more dominant cracks with much more extended cracking off the impact axis in proximity to the specimen boundaries. The simulations also capture cracks appearing on the opposing edge of the specimen, Figures 8(d) and 8(e).

As in the case of the lower velocity, the extent of the damage front was 


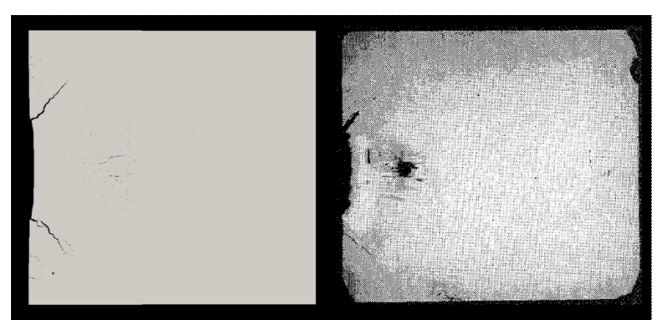

(a) $t=6 \mu \mathrm{s}$

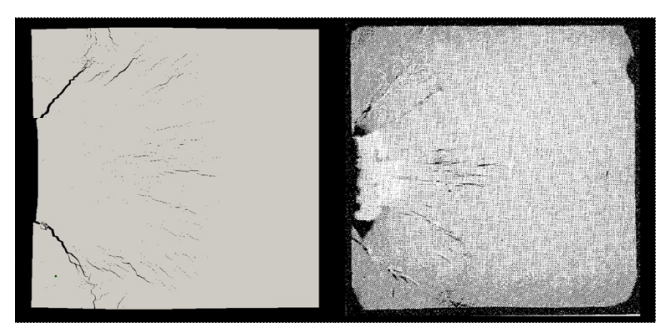

(c) $t=10 \mu \mathrm{s}$

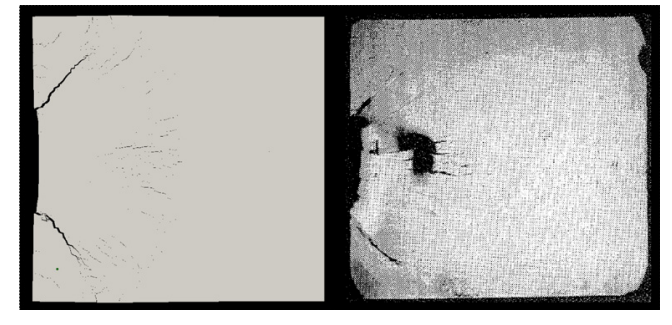

(b) $t=8 \mu \mathrm{s}$

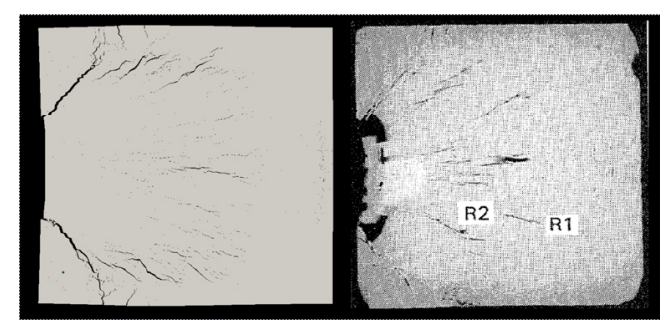

(d) $t=12 \mu \mathrm{s}$

Figure 6: Comparison of simulated (left) and experimental (right) crack propagation patterns for $85 \mathrm{~m} \cdot \mathrm{s}^{-1}$ impact 


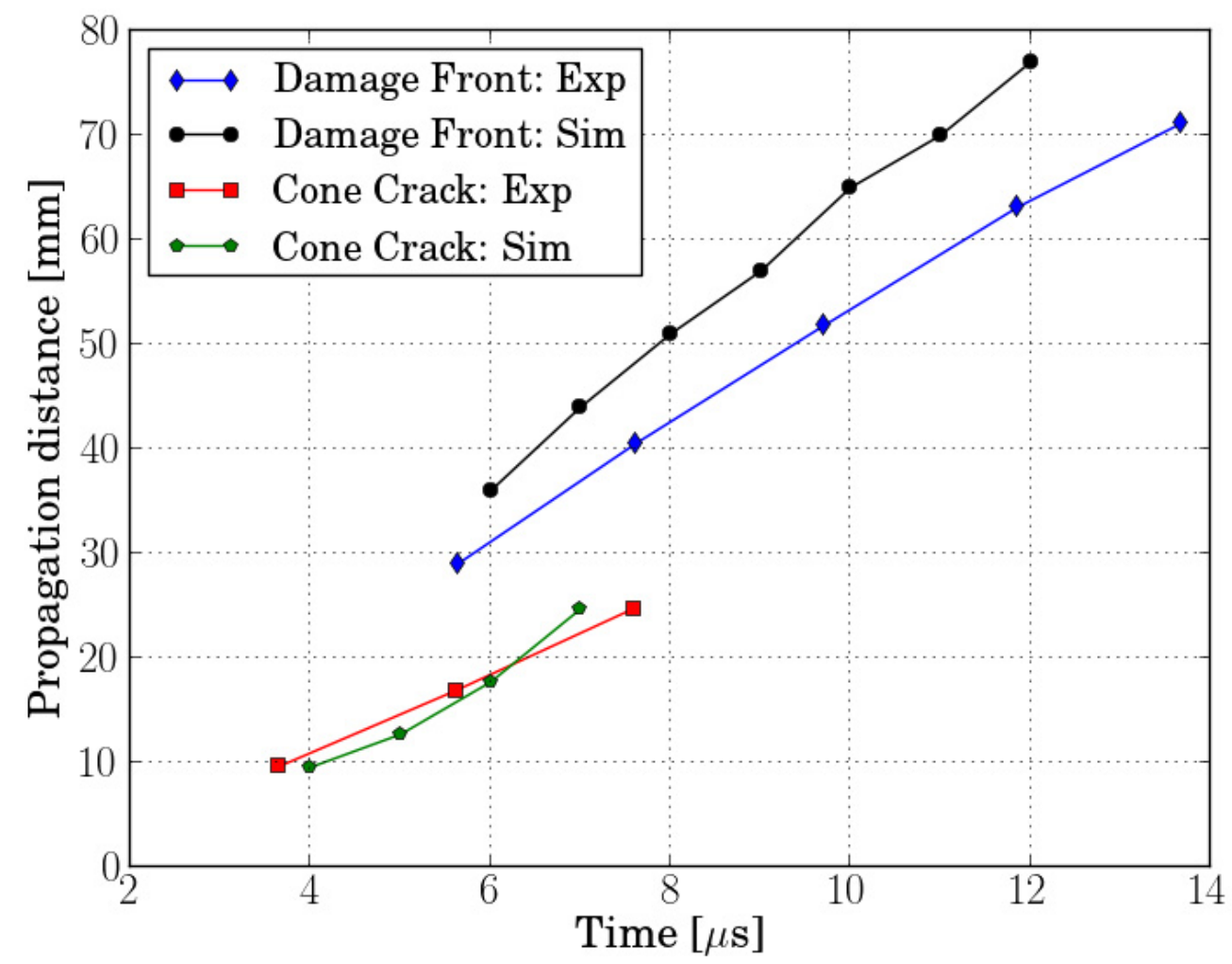

Figure 7: Comparison of simulated and experimental crack propagation lengths over time for the cone crack and the damage front for an impact speed of $85 \mathrm{~m} \cdot \mathrm{s}^{-1}$ 
measured along the centerline of the specimen and compared to the experimentally reported values, Figure 9. Overall, the simulated and experimental damage front locations are in reasonable agreement considering the uncertainty in the material parameters used in the simulations and in the experimental measurements. However, the average speed of the simulated damage front was $7600 \mathrm{~m} \cdot \mathrm{s}^{-1}$ which is significantly higher than the average experimental speed of $5635 \mathrm{~m} \cdot \mathrm{s}^{-1}$ reported in [29].

Using shadowgraph analysis, it has been shown that shear waves drive the nucleation and propagation of damage fronts in EOI experiments on glass plates [29]. In this regard, it is interesting to use the full field description provided by the simulations to confirm and further refine explanations for crack pattern formation based on experimental results. To this end, in Figures 10(a)-10(d) we examine the evolution of the maximum principal stress and its direction from the simulations.

In the early stage of damage formation (Figures 10(a) and 10(b)) the incident longitudinal wave propagates first from the impact site, forming a strong compressive front. In its wake, a complex region of tensile stress forms due to the interaction of shear waves originating from the upper and lower edges of the contact zone and from the reflection of the leading longitudinal wave at the lateral specimen boundaries. A key feature of the shear waves revealed by the simulation results is that their propagation and subsequent interaction leads to strong tensile fronts with semi-circular shapes (see Figures 10(b) and 10(c)). As these fronts propagate along the plate, they expand radially and the maximum tensile stress aligns at each point approximately with the front tangent. Since a traction-based fracture criterion is 


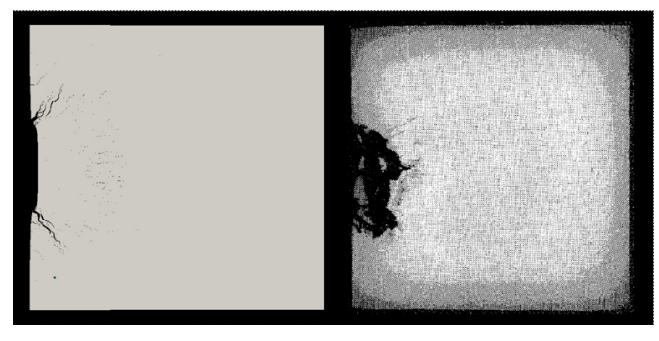

(a) $t=6 \mu \mathrm{s}$

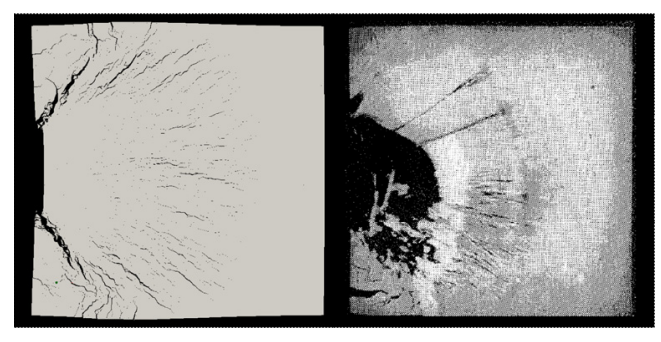

(c) $t=11 \mu \mathrm{s}$

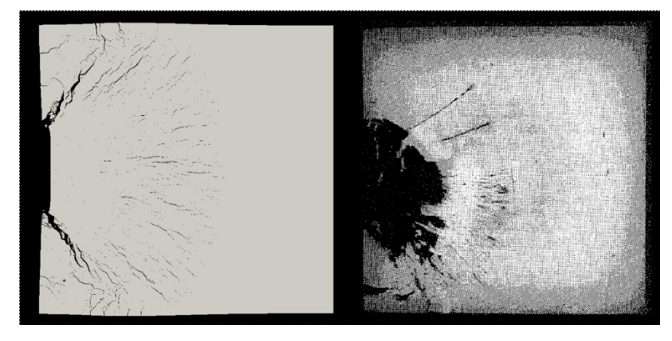

(b) $t=9 \mu \mathrm{s}$

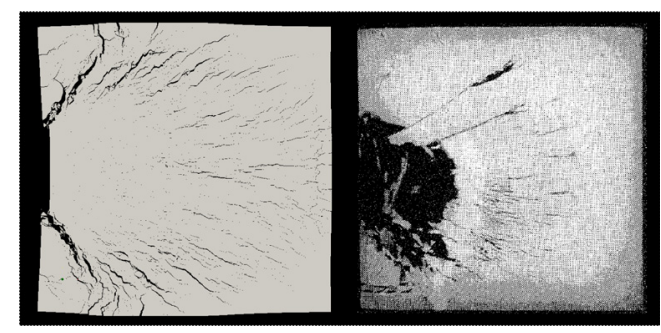

(d) $t=13 \mu \mathrm{s}$

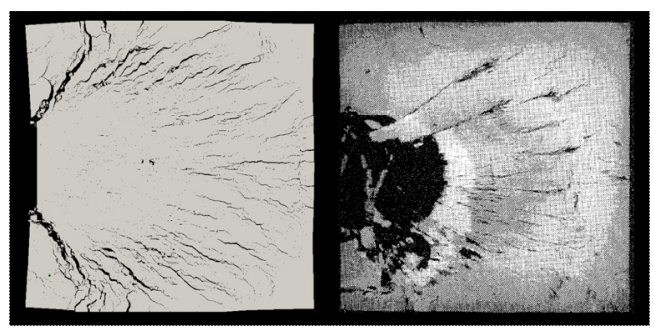

(e) $t=15 \mu \mathrm{s}$

Figure 8: Comparison of simulated (left) and experimental (right) crack propagation patterns for $150 \mathrm{~m} \cdot \mathrm{s}^{-1}$ impact 


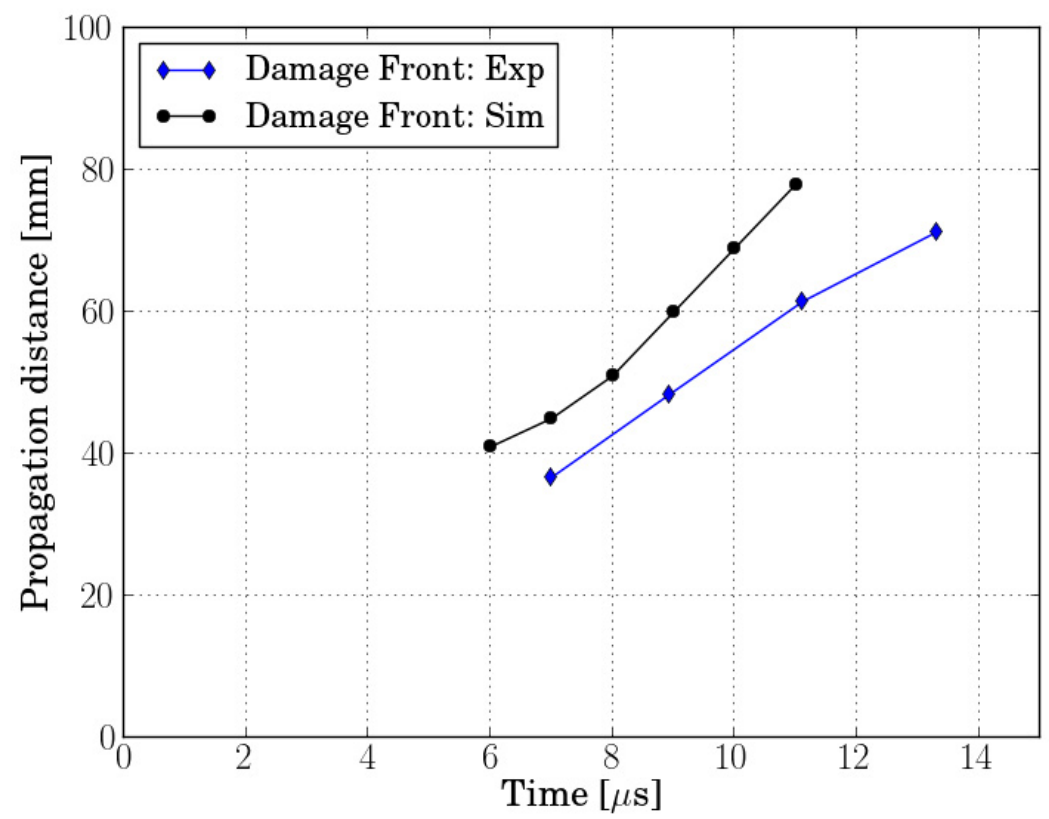

Figure 9: Comparison of the average radial location of the damage front over time from simulations and experiments for an impact velocity of $150 \mathrm{~m} \cdot \mathrm{s}^{-1}$ 
utilized in the simulations, cracks nucleate in radial planes perpendicular to the maximum principal stress direction. Thus, the simulations confirm that the formation of radial damage fronts in EOI experiments is controlled by the evolution of the maximum principal stress resulting from the propagation of shear waves. In Figures 10(b), 10(c), and 10(d) the simulations also show that when the shear waves reach the upper and lower specimen edges, the maximum principal stresses are aligned parallel to the edges. This causes the localized nucleation of cracks at the boundaries which propagate nearly perpendicular to the specimen edges.

Comparing the response predicted at 85 and $150 \mathrm{~m} \cdot \mathrm{s}^{-1}$, we find that the evolution of the maximum principal stress distribution and its direction are similar. However, one key difference is that tensile stresses are substantially stronger at locations away from the centerline of the impactor for the higher velocity case. This drives the nucleation of more cracks in the expanding radial damage front, many of which do not propagate far before arresting, which results in a profusion of short cracks. This crack arrest can be explained by the larger crack density which leads to a fast release of the driving force for crack growth. This explains why as the impact velocity increases there is a transition from the propagation of a few dominant cracks to the propagation of many shorter and more closely-spaced cracks.

\subsection{Normal Impact Simulations}

In this section we simulate the failure of monolithic $\mathrm{Al}_{2} \mathrm{O}_{3}$ plates subjected to normal impact by spherical projectiles. The main goal of this study is to investigate the dependence of fracture patterns on impact velocity using the full-field description provided by the simulations. This complements experi- 


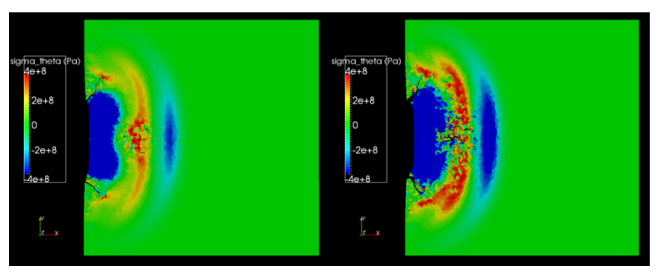

(a) $t=4 \mu \mathrm{s}$

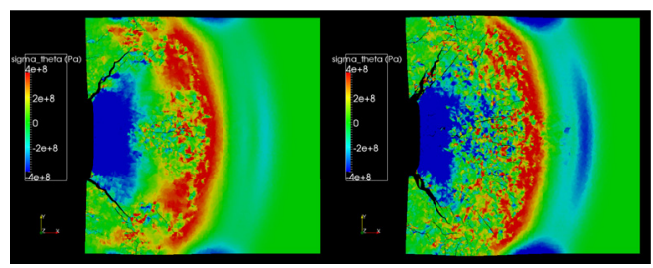

(c) $t=8 \mu \mathrm{s}$

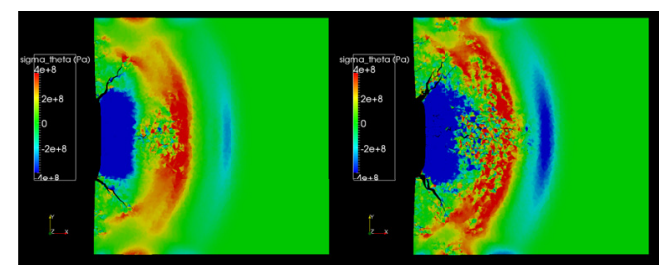

(b) $t=6 \mu \mathrm{s}$

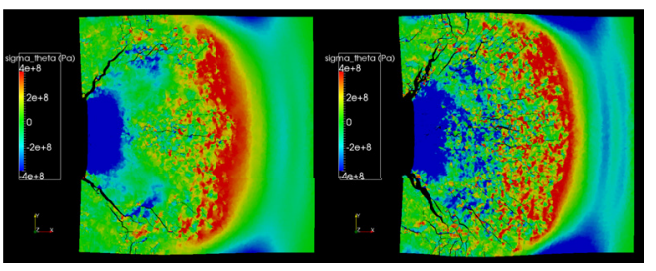

(d) $t=10 \mu \mathrm{s}$

Figure 10: Evolution of the simulated maximum principal stress and associated principal direction. For each time snapshot, the results for the lower impact velocity $\left(85 \mathrm{~m} \cdot \mathrm{s}^{-1}\right)$, shown on the left of the figure, are compared to those for the higher impact velocity (150 $\left.\mathrm{m} \cdot \mathrm{s}^{-1}\right)$, shown on the right of the figure 
mental studies of normal impact on ceramic plates $[3,10,13,14,15,16,17]$. In general, experimental configurations for this type of experiments involve different degrees of confinement of the ceramic plate with a backing and lateral frame for two main reasons: 1) to keep the sample in place and facilitate recovery and post mortem analysis, and 2) because practical uses of hard brittle plates in ceramic armor are known to have a much increased performance under confinement conditions. The effect of different type of boundary conditions provided by different types of confinement on the response of ceramic plate impact including the case of no confinement was investigated by Sherman, [14]. The effect of the confinement usually results in a more complex response. In this work, we focus on the impact response of unconfined and unsupported plates in order to decouple the fracture response of the plate with the complexities of the interaction with the confinement system.

We conducted simulations of the impact of spherical steel projectile at three different velocities spanning the range $100-700 \mathrm{~m} \cdot \mathrm{s}^{-1}$. The projectile radius $(r=3.7 \mathrm{~mm})$ is small compared to the length of the plate $(l=100 \mathrm{~mm})$ leading to a localized impact. The resulting range of impact energies are representative of experimental studies employing metallic projectiles (see e.g. $[3,10,13,14,15,16,17,20])$. Since the upper range of velocities considered here greatly exceeds particle velocities observed at the Hugoniot elastic limit for $\mathrm{Al}_{2} \mathrm{O}_{3}$ [39], we account for the possibility of inelastic deformation by employing the plasticity model for $\mathrm{Al}_{2} \mathrm{O}_{3}$ proposed by Deshpande and co-workers [20]. Details on the model and its parameters are provided in Appendix A.

The finite element mesh was obtained from ABAQUS CAE software using 
an advancing front algorithm. In order to enhance the randomness of the

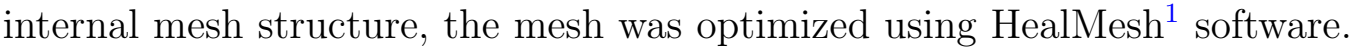
The final finite element mesh used in calculations had an average element size of approximately $800 \mu \mathrm{m}$ and comprised 860,000 tetrahedra (corresponding to 25.8 million degrees of freedom). The material properties and geometry of the plate and projectile are provided in Table A.2 in Appendix A. The spherical projectile was modeled as a rigid body but considering its dynamic response using a mass density corresponding to steel.

Figures 11-13 show predicted cracking patterns for the three impact velocities after a simulation time of approximately $18 \mu \mathrm{s}$. The response for the lowest velocity, Figure 11, shows four radial cracks on the back face of the tile which were arrested before reaching the specimen edges. The lack of any visible cracking on the top surface of the specimen shows that these cracks did not propagate through the thickness. The nearly perpendicular arrangement of the four radial cracks is similar to the "cruciform" type cracking patterns which have been the subject of several prior analytical studies, e.g. $[40,41]$. Besides radial cracking, no other fracture mode is observed.

At the intermediate velocity approximately seven radial cracks are observed on the back face, Figure 12. The increased driving force also activates additional failure modes including the formation of circumferentially oriented cracks on the top surface of the specimen. This fracture mode closely resembles nested ring cracking patterns observed in previous impact experiments on ceramics and glasses, e.g. Figure 2. The lack of much visible ring cracking

\footnotetext{
${ }^{1}$ HealMesh (C) Parasim Inc.
} 
on the back surface of the tile suggests that the ring cracks are localized at the top surface, which has also been observed experimentally in some cases $[2,11,12]$. A fragmented zone with a conical shape is also observed in a localized region under the impactor.

At the highest impact velocity, Figure 13, the predicted cracking patterns share similar features with those for the intermediate velocity, including the formation of a fragmented zone under the impactor and the propagation of radial cracks. In contrast to the intermediate velocity, however, the predicted long range damage is significantly different. Instead of ring cracks, a nonaxisymmetric fracture mode is visible on both the front and back surfaces of the tile in which cracks nucleate in a "box-shaped" pattern. Localized cracking is also observed normal to the specimen boundaries. These localized boundary cracks intersect with the specimen edges on one end and coalesce with the box-shaped cracks on the other end leading to the formation of rectangular fragments at the specimen boundary.

Recent high velocity sphere impact experiments by Compton and Zok on unconfined $\mathrm{Al}_{2} \mathrm{O}_{3}$ tiles [22] exhibit fracture and fragmentation features that are similar to the simulation results presented here for the highest impact velocity. Specifically, Figure 14 shows the post-mortem fracture patterns in a recovered $\mathrm{Al}_{2} \mathrm{O}_{3}$ specimen which was impacted at $1052 \mathrm{~m} \cdot \mathrm{s}^{-1}$ by a 304 stainless steel sphere and supported by a $2 \mathrm{~mm}$ steel backing plate. The experiments show the same fracture modes observed in the simulation, including a localized fragmented zone under the impactor, radial cracks, a box-shaped cracking pattern, and localized cracking at the specimen edges which results in rectangular fragments. 


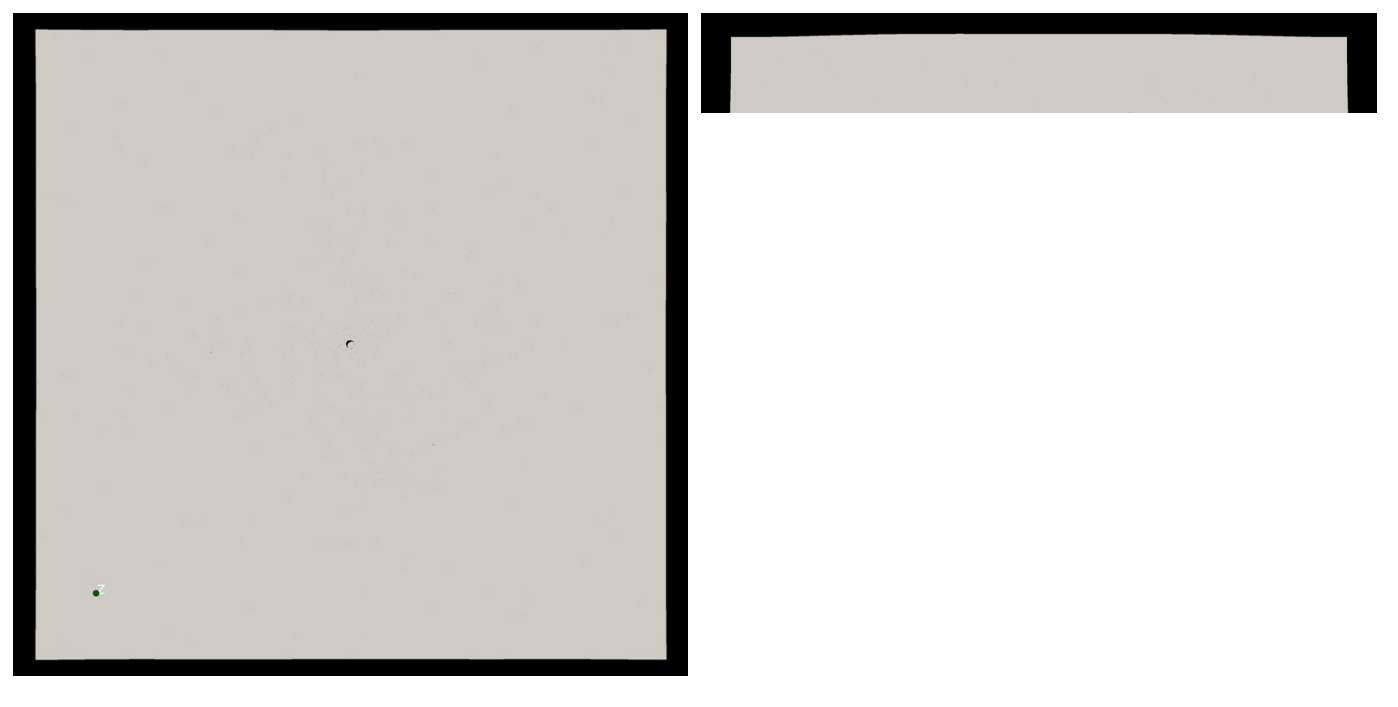

